\title{
THE TOXICITY OF EPICHLORHYDRIN VAPOUR
}

\author{
BY \\ J. C. GAGE \\ From the Imperial Chemical Industries Limited, Industrial Hygiene Research Laboratories
}

(RECEIVED FOR PUBLICATION JUNE 2, 1958)

\begin{abstract}
A versatile apparatus is described for exposing experimental animals to predetermined concentrations of toxic substances in air, and it has been used to study the vapour toxicity of epichlorhydrin to rats and rabbits. At concentrations above 100 p.p.m. the vapour produces lung oedema and damage to the renal cortical tubules in rats, while above 50 p.p.m. it produces marked nasal irritation. Nasal irritation is still present in rats and rabbits below 50 p.p.m. and is only absent below 10 p.p.m. It is recommended that the concentration in industrial atmospheres should not exceed 5 p.p.m.
\end{abstract}

Epichlorhydrin is a colourless liquid with a chloroform-like odour; its boiling point is $116^{\circ} \mathrm{C}$., density 1.18 at $20^{\circ} \mathrm{C}$. and vapour pressure $13 \mathrm{~mm}$. at $20^{\circ} \mathrm{C}$. It has the following structure

$\mathrm{CH}_{2} \cdot \mathrm{CH} \cdot \mathrm{CH}_{2} \cdot \mathrm{Cl}$ and its high chemical reactivity is due both to the epoxy group and to the chlorine atom. It is used as a stabilizer in certain chlorinecontaining substances such as pesticides and chlorinated rubber and as an intermediate in many manufactures. Epichlorhydrin is finding an increasing outlet in the production of epoxy resins for surface coatings, castings, laminates, and adhesives.

There is not an extensive literature on the toxicity of epichlorhydrin, although some reference is made in the literature of one manufacturer to an experimental investigation (Freuder and Leake, 1941) which states that the liquid is a vesicant and a dermatitic agent to the skin, and that the vapour is irritating to the eyes, nose, and throat. It has been shown that the exposure of rats to an atmospheric concentration of 250 p.p.m. for four hours is lethal (Carpenter, Smyth, and Pozzani, 1949), but no maximal allowable concentration is recommended in any available published list. The present investigation was undertaken to ascertain the threshold of the toxic action of epichlorhydrin vapour on experimental animals, so that a limiting concentration for industrial atmospheres might be assessed.

Design of Exposure Chamber

The chamber in which the animals were exposed to test epichlorhydrin atmospheres is shown in Figs. 1 and 2.
The animals were housed in the " perspex" exposure chamber, $A$, which stood in an outer glass-walled chamber, $B$, maintained at a slightly reduced pressure by means of an exhaust fan. The test atmosphere was led into the bottom of the exposure chamber through the glass column, $D$, and left the chamber through a series of holes near the base.

Generation of Test Atmospheres.-The epichlorhydrin atmospheres were prepared by atomizing solutions of epichlorhydrin in propanol into a metered stream of air by means of a controlled fluid-feed atomizer (Gage, 1953). This is shown at $C$ in Fig. 2 and differs from the model previously described in having the atomizer fused directly to the glass syringe to prevent leakage. The piston of the syringe was lubricated with a glyceroldextrin-mannitol grease (Meloche and Fredrick, 1932), which has been found to be much less readily attacked by organic solvents than other available lubricants. The atomizer assembly was clamped vertically with its tip penetrating a central hole in the sponge rubber seating $E$ of the glass column $D$. The syringe was driven by a slow injection apparatus $F(C$. F. Palmer, Ltd.) which has been modified by the makers to be suitable for a vertical drive. This mechanism operates a ram by means of a synchronous motor through a set of gears which can drive the syringe piston at speeds of one inch in $10,20,40,80,160$, or 320 minutes. The air supply to the atomizer was controlled by the valves $G, H$, and with a pressure gauge, $I$, shown on the right of the instrument panel in Fig. 1 and also displayed diagrammatically on the right of Fig. 3. A common compressed air-line for a block of three exposure chambers, controlled by a "nullmatic" pressure regulator (Sunvic Controls, Ltd.) brought air at a constant pressure of $15 \mathrm{lb}$. per square inch to valve $G$ and thence to the needle valve $H$.

From $H$ the air passed to the atomizer $C$, with a teepiece for the pressure gauge $I$. At least once a week the calibration of the pressure gauge in terms of air-flow rate 





was checked by attaching a rotameter to the outlet of the atomizer. This system of flow control by needle valve and pressure gauge with a constant air-line pressure has been found to be simpler and at least as satisfactory as an individual pressure reducing valve and flow meter on each exposure chamber. The concentration of epichlorhydrin in the test atmosphere was calculated from the internal diameter of the syringe, the rate of movement of the piston, the concentration of epichlorhydrin in solution, and the air flow supplied to the atomizer.

Exposure Chamber.-The exposure chamber $A$ was constructed from four perspex walls, $47 \times 47 \times 30 \mathrm{~cm}$. high, with a detachable lid. The open bottom rested on an aluminium tray, $J$, and the animals were placed on a grid $K$ constructed from stainless steel wire. The upper end of an aluminium collar $L$, which had a diameter large enough to take the glass column $D$, passed through a hole in the centre of grid $K$, and the narrower lower end passed through a smaller hole in the tray $J$ and through another in the aluminium base of the outer chamber $B$. The glass column $D$ was sealed into $L$ by means of a sponge rubber pad $E$, which also carried a hole through which passed the tip of the atomizer $C$. The animals in the chamber were kept apart by means of radial partitions, eight of which were inserted on stainless steel supports round the centre glass column, and a second stainless steel grid was placed on top of these partitions to prevent the animals from climbing over them.

Analysis of Test Atmosphere.-A daily analytical check on the concentration of the epichlorhydrin atmospheres was made by the method described by Daniel and Gage (1956). For this purpose a sampling port was provided in the side of the exposure chamber, kept closed when not in use by means of a rubber stopper. The apparatus for drawing the required air sample through the absorber is shown on the left of Figs. 1 and 3. The sample of the test atmosphere was collected by means of a vacuum line controlled by a critical orifice plate; this provides a sampling rate independent of variations in the vacuum line and determined by the line pressure between the orifice and the absorber. The orifice plate can be seen at $O$ in Fig. 3, screwed in its holder between two rubber gaskets; for sampling speeds of 0.5 to $1.01 . / \mathrm{min}$. an orifice with a diameter of $0.45 \mathrm{~mm}$., flared on the upstream side to prevent turbulence, is suitable. A vacuum line was led to the orifice through the valve $M$ and applied a vacuum of about $50 \mathrm{~mm}$. mercury to the orifice. Between the sampling equipment and the orifice plate at $O$ was the needle valve $N$ and vacuum gauge $P$. The vacuum gauge was calibrated in terms of flow rate by replacing the sampling equipment with a suitable rotameter and taking readings of the rotameter and vacuum gauge over a range of settings of the needle valve. A check on this calibration was made at least once a week.

\section{Experimental Results on Rats}

At each test epichlorhydrin concentration four male and four female albino Wistar rats, within the weight range $160-200 \mathrm{~g}$., were exposed to the atmospheres for daily six-hour periods, for five days a week. They were weighed daily and blood samples were taken weekly from the tail vein for haemoglobin determination by the Haldane method and for red and white cell counts and a differential white cell count on a Leishman-stained smear. Each week an 18-hour overnight sample of pooled urine from the female rats was taken for protein estimation by a colorimetric biuret method after trichloroacetic acid precipitation.

Eleven Exposures at 120 p.p.m.-After three hours of the first exposure all rats showed laboured breathing which remained throughout the subsequent exposure periods. Between the exposures they were lethargic and in progressively poor condition. After the fourth exposure a profuse nasal discharge developed. The rats showed a considerable loss in weight during the exposure and there was a marked leucocytosis due to an increase in both polymorphs and monocytes. At the termination of the exposures the urinary protein excretion was more than double the normal value. One rat died after the eleventh exposure.

At necropsy all lungs showed patches of discoloration, two livers had uneven coloration, and in two rats the kidney cortex was unusually pale. The intestines contained bile and were distended with gas. At histopathological examination the lungs of all rats showed congestion, oedema, consolidation, and areas of inflammation with signs of abscess formation. There was general congestion of the liver, in one case accompanied by areas of necrosis. The kidneys of all rats showed areas of leucocytic reaction and in four there was atrophy of the peripheral cortical tubules.

Eighteen Exposures at 56 p.p.m.-After the tenth exposure the rats appeared to be lethargic with some respiratory distress; later a nasal discharge appeared. The rats lost weight during the exposure days but recovered to some extent during the week-ends. The urinary protein excretion, haemoglobin, and red, white, and differential cell counts were within normal limits. No abnormalities were observed at necropsy or on histopathological examination, apart from abscess formation in one lung which was probably not attributable to the exposure.

Eighteen Exposures at 27 p.p.m.-Signs of mild nasal irritation were produced by this concentration, and the body weight remained constant during the exposure period. The lung of one rat at histopathological examination showed areas of haemorrhage and consolidation.

Nineteen Exposures at 17 p.p.m.-This group of rats gained weight normally though their condition 
appeared somewhat inferior to that normal for this colony. Nothing abnormal was observed at necropsy or at histopathological examination.

Eighteen Exposures at 9 p.p.m.- Apart from two rats in this group which developed a pulmonary infection, the rats grew normally and appeared in good condition.

\section{Experimental Results on Rabbits}

The effect of lower epichlorhydrin concentrations has been tested on New Zealand white male rabbits within the weight range 1.8 to $2 \mathrm{~kg}$. Two rabbits, exposed to 35 p.p.m. for 20 daily periods, showed signs of nasal irritation but increased in weight normally. Two more rabbits were exposed to 16 p.p.m. and also showed nasal irritation after two exposures; the concentration was decreased to 9 p.p.m. and then continued for 20 days without producing effects on the rabbits. Post-mortem and histopathological examination of these rabbits at the end of the exposure period showed no signs of any tissue changes which could be attributed to the exposures.

\section{Discussion}

A wide variety of exposure chambers are described in the literature, but the design used in this investigation has novel features which have been evolved during experience over a number of years. The "perspex" chamber gives complete visibility and is easy to clean and the outer casing prevents contamination of the laboratory atmosphere. This type of construction is less expensive and easier to operate than a sealed metal and glass chamber. The procedure for generating the test atmosphere has certain advantages over the more usual methods of bubbling the air through a volatile liquid, or metering the liquid on to a hot surface in an air stream. By the method described known concentrations can be prepared without knowledge of the physical constants of the substance and these concentrations can be varied at will, and impure samples or mixtures can be examined without a progressive change in the atmosphere during the experiment. The substance is completely volatilized into the air stream without the application of heat and the bulk is not exposed to continuous aeration with the attendant possibility of oxidation, polymerization, or other chemical changes. If a solution of a non-volatile liquid or solid in an inert volatile solvent is used in the syringe, a fine mist or dust is produced, and low dilutions of a gas can be prepared by directly injecting the gas into the air stream by means of a large glass syringe.

The results obtained with epichlorhydrin vapour on rats indicate that above 50 p.p.m. it produces a marked nasal irritation, and above 100 p.p.m. it can give rise to pulmonary oedema together with damage to the renal cortical tubules. There can be little doubt that this toxic effect is due to the chemical action of the epoxide group which is rendered more active by the presence of the chlorine atom, as epichlorhydrin is much more toxic than ethylene oxide and propylene oxide (Hollingsworth, Rowe, Oyen, McCollister, and Spencer, 1956). It is well known that the epoxide ring can react with anions to form esters and it seems probable that modification of cell proteins by esterification of carboxyl groups gives rise to a change in function which recalls the effects produced by phosgene and ketene, which have been attributed to the acetylating action of these substances on tissue proteins (Potts, Simon, and Gerard, 1949).

The experiments on rats and rabbits have shown that nasal irritation is still apparent below 50 p.p.m. epichlorhydrin and under the experimental conditions is only absent below 10 p.p.m. In the rat experiments there was some evidence of a higher incidence of lung infections than is encountered when rats of this colony are maintained in the exposure chamber, and although this may be fortuitous, the possibility that the inhalation of the vapour can predispose to infection should not be ignored. It is recommended that the concentration of epichlorhydrin in the atmosphere where men are continually working should not exceed 5 p.p.m. unless respiratory protection is used. This concentration can readily be determined in air (Daniel and Gage, 1956).

Technical assistance in this investigation was supplied by Mr. Z. S. Bérczy, and I am indebted to Dr. J. G. S. Crabbe for a pathological report on the histological preparations. The exposure chambers were constructed by the Engineering Department of the I.C.I. Akers Research Laboratories.

\section{REFERENCES}

Carpenter, C. P., Smyth, H. F., and Pozzani, U. C. (1949). J. industr. Hyg., 31, 343.

Daniel, J. .̈.,, and Gage, J. C. (1956). Analyst, 81, 594.

Freuder, E., and Leake, C. D. (1941). Univ. Calif. Publ. Pharmacol. 2, 69. [Quoted by Shell Chemical Corp., Technical Booklet SC : 46-3 "Epichlorhydrin ", 1946.]

Gage, J. C. (1953). J. sci. Instrum., 30, 25. and Spencer, H. C. (1956). A.M.A. Arch. industr. Hlth, 13, 217.

Meloche, $\dot{C}$. C., and Fredrick, W. G. (1932). J. Amer. chem. Soc.,

Potts, A. M., Simon, F. P., and Gerard, R. W. (1949). Arch. Biochem., 24, 329. 